\title{
Percepção de uma população sobre o contágio da gripe pelo vírus Influenza A - H1N1
}

RESUMO | Objetivo: Estudo investigativo que avaliou o conhecimento acerca da transmissão, prevenção e controle da Influenza A - H1N1. Método: Foi realizada uma pesquisa descritiva, exploratória e quantitativa, junto à população da cidade de Uberaba/ MG, no período de 01/10/2018 a 21/10/2018, onde se respondeu a um questionário em que se tratava de questões pertinentes ao conhecimento geral, contágio, tratamento e cura da doença causada pelo H1N1, foram determinadas as frequências para cada resposta e avaliadas por teste de hipótese (Q-Quadrado). Resultados: Um índice baixo de conhecimento geral foi encontrado (78\%), seguido de $82 \%$ de respostas incorretas quanto ao tratamento e a cura, e de $59 \%$ de respostas incorretas sobre questões referentes às formas de contágio da doença. Conclusão: Os dados contribuem para demonstrar divergência entre os mecanismos traçadores da geração em Educação em Saúde, apontando assim, para necessidade de um melhor direcionamento na prática da manutenção e geração à saúde pública.

Palavras-chaves: Educação em Saúde; influenza A; Promoção da Saúde.

\begin{abstract}
Objective: An investigative study that evaluated the knowledge about the transmission, prevention and control of Influenza A - H1N1. Method: A descriptive, exploratory and quantitative research was carried out, together with the population of the city of Uberaba / MG, from 01/10/2018 to 21/10/2018, where a questionnaire was answered in which they were pertinent questions to the general knowledge, contagion, treatment and cure of the disease caused by $\mathrm{H} 1 \mathrm{~N} 1$, the frequencies were determined for each response and evaluated by hypothesis test (Q-Square). Results: A low general knowledge index was found (78\%), followed by $82 \%$ of incorrect answers regarding treatment and cure, and $59 \%$ of incorrect answers on questions regarding the forms of disease contagion. Conclusion: The data contribute to demonstrate divergence between the tracing mechanisms of generation in Health Education, thus pointing to the need for a better orientation in the practice of maintenance and generation to public health.
\end{abstract}

Keywords: Health Education; influenza A; Health promotion.

RESUMEN | Objetivo: Estudio investigativo que evaluó el conocimiento acerca de la transmisión, prevención y control de la Influenza A - H1N1. Método: Se realizó una investigación descriptiva, exploratoria y cuantitativa, junto a la población de la ciudad de Uberaba / MG, en el período del 01/10/2018 al 21/10/2018, donde se respondió a un cuestionario en el que se trataba de cuestiones pertinentes el conocimiento, el contagio, el tratamiento y la curación de la enfermedad causada por el H1N1, se determinaron las frecuencias para cada respuesta y evaluadas por prueba de hipótesis (Q-Cuadrado). Resultados: Un índice bajo de conocimiento general fue encontrado (78\%), seguido de $82 \%$ de respuestas incorrectas en cuanto al tratamiento y la cura, y del $59 \%$ de respuestas incorrectas sobre cuestiones referentes a las formas de contagio de la enfermedad. Conclusión: Los datos contribuyen a demostrar divergencia entre los mecanismos trazadores de la generación en Educación en Salud, apuntando así, para necesidad de un mejor direccionamiento en la práctica del mantenimiento y generación a la salud pública.

Palavras claves: Educación en Salud; influenza A; Promoción de la Salud.

\section{Camila Botelho Miguel}

Biomédica, especialista em Biologia Celular e Molecular, Mestre em Patologia Clínica, Doutora e Pós-doutorado em Ciências da Saúde. Universidade Federal do Triângulo Mineiro (UFTM), Uberaba, MG, Brasil; Centro Universitário de Mineiros (UNIFIMES), Mineiros, GO, Brasil.

\section{Wladimir Rodrigues Faustino}

Enfermeiro, Especialista em UTI Adulto, Neonatologia, Pediatria e Nefrologia, Mestre em Enfermagem Profissional na Gênese do processo do cuidar em Enfermagem. Faculdade do Norte de Mato Grosso (AJES), Guarantã do Norte, MT, Brasil.

\section{Fabiana Rezer}

Enfermeira, Especialista em Nefrologia e Mestre Profissional em Enfermagem no Processo do Cuidar em Saúde. Faculdade do Norte de Mato Grosso (AJES), Guarantã do Norte, MT, Brasil.

\section{Guilherme Ferretto}

Enfermeiro, Especialista em Enfermagem do Trabalho e Urgência e Emergência. Centro de Educação Profissional CEFORES/UFTM, Uberaba, MG, Brasil.

Recebido em: 21/01/2019

Aprovado em: 16/06/2019

\section{Carlo José Freire Oliveira}

Graduado em Medicina Veterinária, Especialista em docência no ensino superior, aperfeiçoamento em educação integral, Mestrado, Doutorado e Pósdoutorado em Imunologia Básica e Aplicada. Universidade Federal do Triângulo Mineiro (UFTM), Uberaba, MG, Brasil.

\section{Wellington Francisco Rodrigues}

Graduação em Ciências Biológicas e Biomedicina, Especialização em Biologia Celular e Molecular, Especialização em Imunologia Clínica e Laboratorial, Mestrado em Odontologia em ênfase em Biopatologia, Doutorado em Genética e Bioquímica e Doutorado em Ciências da Saúde. Universidade Federal do Triângulo Mineiro (UFTM), Uberaba, MG, Brasil. 
Introdução

$\mathrm{D}$ oenças virais estão associadas em rápida disseminação e capacidade de geração de doenças severas. A gripe é uma doença infecto contagiosa provocada pelo vírus da Influenza. Uma nova mutação do referido vírus gerou a Influenza A, hemaglutinina de tipo 1 e neuraminidase do tipo 1 (H1N1), da qual foi capaz de provocar uma pandemia, e traz preocupações quanto ao preparo populacional em relação à novas pandemias. ${ }^{1-3}$ Embora sua patogenicidade seja semelhante às subespécies da Influenza A, o H1N1 é capaz de provocar uma estimulação exacerbada do sistema imune com a liberação de mediadores inflamatórios, histaminas e prostaglandinas no local de invasão e aparelho respiratório, favorecendo a proliferação de microrganismos como bactérias e fungos, podendo levar a morte do hospedeiro. ${ }^{1,2,4-6}$

No Brasil, entre abril de 2009 e março de 2010, foram confirmados 46.355 casos de síndrome respiratória aguda grave, em decorrência da infecção pelo vírus da Influenza A. ${ }^{1,2,4-6}$ Embora se tenha um tratamento que visa diminuir a carga viral, a melhor forma de se conter a progressão e disseminação do vírus é por meio da prevenção, lavagem das mãos, proteção ao tossir e ou espirrar, uma vez que o contágio está diretamente relacionado com a proliferação de partículas virais contidas em gotículas de secreções respiratórias de indivíduos infectados e pela presença do vírus no ambiente. A prática da Educação em Saúde se tornou uma grande aliada na prevenção de diversas doenças, facilitando a compreensão de fatores associados com a doença e favorecendo a prevenção. ${ }^{7-8-9}$

Em 2009, a Organização Mundial de Saúde (OMS) elevou ao máximo o nível de alerta pandêmico global, devido à rápida propagação de um novo tipo de Influenza, o H1N1, a partir da linhagem suína classificada como A/California/7/2009 H1N13 10. O motivo do comunicado de emergência em Saúde Pública foi a alta abrangência da doença, com evidência de transmissão comunitária disseminada em pelo menos dois continentes, tendo em vista a manutenção de baixa letalidade. ${ }^{11}$

A Influenza A - H1N1 é uma doença de distribuição universal, com ocorrência sazonal ou endêmica. Entretanto, devido à capacidade de mutações do vírus, a população pode entrar em contato com novas formas variantes, com risco de haver novas epidemias ou pandemia no futuro. ${ }^{12}$

As campanhas anuais de vacinação vêm sendo consideradas estratégias de sucesso contra a doença, tendo em vista a ampla adesão das populações alvo à iniciativa. ${ }^{13}$ Além disso, este tema tem ganhado maior importância nos últimos meses devido ao surgimento de novos casos no Brasil, sendo que já houve mais de 100 mortes no ano de 2016 e há grande possibilidade de aumento desse número ao longo do ano, especialmente nos grupos de risco que incluem crianças de seis meses a menores de 5 anos de idade, gestantes e idosos com mais de 60 anos.

Ao findar o surto da doença e as campanhas anuais de vacinação, novas variantes virais passam a circular entre a população. Por esse motivo, justifica-se a necessidade do pleno conhecimento sobre a doença. ${ }^{14} \mathrm{O}$ Ministério da Saúde recomenda atualização epidemiológica aos gestores de saúde, capacitação técnica aos profissionais de saúde, e à população recomenda-se a informação sobre os riscos de transmissão e meios deprevenção da doença. ${ }^{15} \mathrm{O}$ conhecimento coletivo é um dos aspectos mais importantes para o direcionamento das ações de saúde, pois determina o comportamento social básico. ${ }^{16}$

Segundo alguns autores, a população necessita do conhecimento para aumentar sua autonomia nos cuidados, individuais e coletivos. ${ }^{17}$ Para tanto, é importante compreender as lacunas de conhecimento da população sobre riscos e agravos existentes. ${ }^{18}$

O conhecimento em saúde é um dos fatores que determinam a vulnerabilidade do indivíduo ao risco de adoecer. ${ }^{19}$ Desse modo, propõem-se uma estrutura de aná- lise que incorpora a auto-avaliação, por meio de questionamento, para se verificar os saberes e as práticas, o status social e o acesso à informação, avaliando-se como pode ocorrer a transmissão de uma doença.

Diante disso, foi proposto um estudo investigativo em um município da região do Triângulo Mineiro, objetivando descrever e avaliar a percepção da população local, acerca da transmissão, prevenção e controle da Influenza A - H1N1, com a seguinte questão norteadora: Qual é o conhecimento da população sobre prevenção, promoção e controle da influenza H1N1?

\section{Método}

Trata-se de uma pesquisa descritiva, exploratória, com abordagem quantitativa. Esse tipo de pesquisa procura estabelecer uma nova visão do problema, classificando e explicando os fatos que ocorrem, sua natureza, características e causas. A pesquisa exploratória tem como finalidade a delimitação do tema a ser pesquisado, a formulação de hipóteses e estabelecimento claro dos objetivos, fornecendo ao pesquisador mais informações sobre o assunto pesquisado ${ }^{19-20}$.

$\mathrm{Na}$ pesquisa quantitativa são utilizados recursos e instrumentos estatísticos (porcentagem, média, mediana, desvio-padrão etc.), necessitando que o pesquisador apresente as hipóteses e variáveis claramente definidas1919,21.

O presente estudo foi executado com uma amostra de 512 indivíduos, em Uberaba-MG, onde foi realizado em diferentes regiões da cidade de forma aleatória; foram percorridos 10 distintos locais com um raio mínimo de $12,8 \mathrm{Km}$, a coleta de dados ocorreu no período de 01/10/2018 a 21/10/2018.

Como critérios de inclusão: indivíduos adultos ( $\geq 18$ anos de idade), munidos de documento de identificação (documentos aceitáveis nacionalmente com foto). Lembrando que a não aceitação em participar da pesquisa não excluiu o indivíduo aos 
benefícios da atividade.

Como critérios de exclusão: não foram consideradas os formulários cuja as questões foram preenchidas indevidamente, com duas ou mais respostas no mesmo item. A não observância deste item poderia ter afetado a análise e refletir nos resultados.

Para a coleta dos dados foi aplicado um questionário semiestruturado com (10 questões), visando abordar a percepção dos pesquisados em relação ao contágio, prevenção e tratamento da gripe causada pelo vírus da Influenza A - H1N1. A idade e o gênero foram inicialmente obtidos, seguido de questões de múltiplas escolhas, como segue: (1) $\mathrm{O}$ que o vírus H1N1 pode causar? (2) Qual a diferença entre a gripe comum e a H1N1? (3) Quais os sintomas associados à gripe $\mathrm{H} 1 \mathrm{~N} 1$ ? (4) Qual a conduta após apresentar sintomas sugestivos à gripe? (5) Qual período que o vírus pode permanecer fora do ambiente corporal? (6) Quais os fatores que se associam com a contaminação? (7) Quais as medidas preventivas ao contágio? (8) Há contraindicações para a vacinação? (9) Há possibilidade de contaminação após a vacinação? (10) Já se vacinou?

Para análise dos dados foram determinadas as frequências para cada resposta, levando em consideração o $\mathrm{N}$-amostral e avaliadas por teste de hipótese (Q-Quadrado), onde foram consideradas as diferenças estatisticamente significantes quando $\mathrm{p}<0,05$.

Todos participantes assinaram o termo de Consentimento livre e Esclarecido (TCLE); todos os procedimentos foram previamente cadastrados na plataforma Brasil e aprovados pelo comitê de ética da Universidade Federal do Triângulo Mineiro (sob o número: 900.025/2014).

\section{Resultados}

As somatórias das respostas foram avaliadas e associadas com um teor de conhecimentos geral baixo/nenhum $(0$ a 4 respostas corretas), médio (5 a 7 respostas corretas) e alto (de 8 a 10 respostas corretas). Para esta avaliação foi verificado um índice elevado para o teor de conhecimento geral baixo/nenhum, com cerca de $78 \%$ da população avaliada. Já para o índice de conhecimento geral médio, encontrou-se um percentual de $13 \%$, acarretando um índice de $9 \%$ para a frequência populacional com um teor elevado de conhecimento geral acerca da gripe causada pelo vírus $\mathrm{H} 1 \mathrm{~N} 1$.

Avaliou-se a frequência de respostas corretas, incorretas e indecisas quanto ao contágio do vírus, um fator importante para a prevenção e disseminação da doença. Cerca de $59 \%$ das afirmativas foram incorretas, seguido de $24 \%$ para indecisos e de apenas $17 \%$ para afirmativas corretas.

Foi avaliado um importante evento após a infecção e desenvolvimento da doença, o tratamento e cura da gripe causada pelo vírus da Influenza $A-H 1 N 1$. Foi evidenciada uma frequência de afirmações incorretas quanto ao conhecimento do tratamento de cura de $83 \%$, seguido de $11 \%$ para afirmações corretas e $7 \%$ para indecisos (tabela 1).

Para se compreender se as frequências negativas estavam relacionadas a alguma faixa etária, os dados foram estratificados quanto aos diferentes grupos etários (18-

\section{Tabela 1. Avaliação descritiva do desfecho indagativo em torno da Gripe por Influenza A - H1N1. Uberaba, MG, Brasil, 2019.}

\section{Frequência relativa das respostas frente aos questionamentos (\%)}

\section{1 - Conhecimento geral sobre a gripe pelo H1N1}

\begin{tabular}{|c|c|c|c|}
\hline & Baixo/Nenhum & Intermediário & Elevado \\
\hline & 78 & 13 & 9 \\
\hline & \multicolumn{3}{|c|}{2 - Questionamentos em relação ao contágio } \\
\hline & 59 & 17 & 24 \\
\hline \multicolumn{4}{|c|}{3 - Indagações sobre tratamento e cura } \\
\hline & 83 & 11 & 7 \\
\hline \multicolumn{4}{|c|}{ Sumarização (2 e 3) } \\
\hline & Incorretas & Corretas & Indecisos \\
\hline Mediana (min-máx) & $78(59-83)$ & $13(7-24)$ & $11(9-17)$ \\
\hline
\end{tabular}


30; 31-50; 51-70 e acima de 70 anos de idade) e aos índices de respostas sim, não é indeciso (Figura 1). Os resultados foram conclusivos para uma variação percentual entre as faixas etárias, porém não significativa estatisticamente ( $p=0,69$ - Qui-quadrado $=3,89$ ).
Embora não houvesse diferenças entre as diferentes faixas etárias, foi verificado se houve uma variação significativa das respostas em relação aos gêneros (Figura 2). Dos 512 participantes avaliados no total, 203 eram do sexo masculino e 309 do sexo feminino. De forma semelhante às faixas
Figura 1. Variações das frequências de afirmações entre diferentes grupos etários, foi testada pelo teste Qui-Quadrado, considerando um a de 5\%. Uberaba, MG, Brasil, 2019.

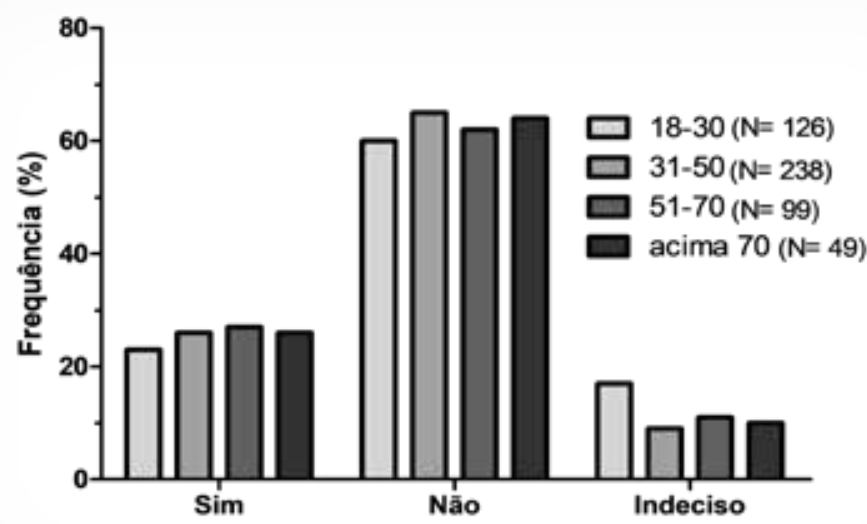

Figura 2. Avaliação da frequência de afirmações entre os diferentes gêneros. A. Opção de tratamento em percentual. B. Sobrevida do Virus em percentual. C. A Contraindicação na vacinação em percentual. A hipótese alternativa que há independência entre os grupos quanto à frequência de respostas foi testada pelo teste Qui-Quadrado, foi considerado um a de 5\%. Uberaba, MG, Brasil, 2019.
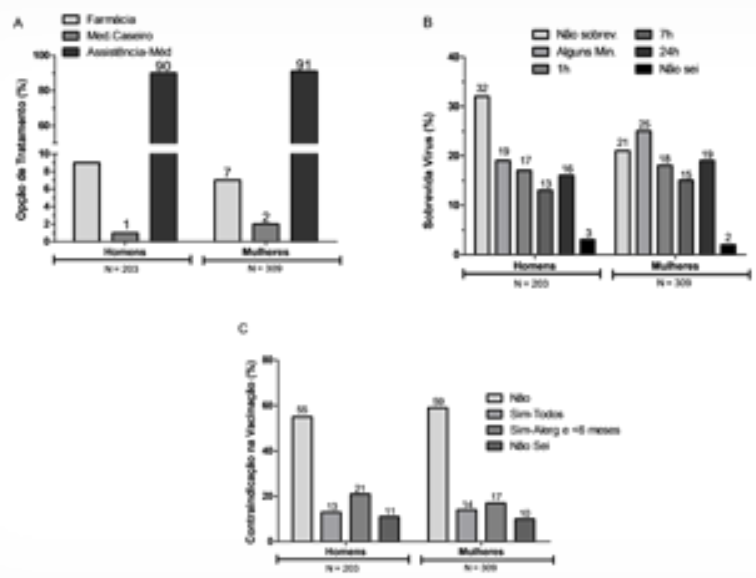

etárias, não foram encontradas diferenças significativas entre os gêneros $(p>0,05)$ nos três itens avaliados: Opção de tratamento (Figura 2a), $\mathrm{p}=0,74$; Sobrevida do vírus (Figura $2 b), p=0,58$ e Contraindicação na vacinação (Figura $2 \mathrm{c}$ ), $\mathrm{p}=0,88$.

\section{Discussão}

De forma geral o conhecimento da população avaliada neste estudo possui um déficit relevante quanto às informações gerais e pontuais dado ao contágio, tratamento e cura da doença, demonstrando assim, um conflito nos objetivos da gestão em saúde pública e os seus resultados. A prática na Educação em Saúde já se mostrou conflitante e contraditória historicamente, pautada em divergências conceituais e políticas, onde os resultados destes conflitos refletem nos objetivos da diminuição com gastos públicos na geração e manutenção à saúde ${ }^{22}$.

O que parece relevante na contextualização à prevenção da doença e manutenção da saúde, é que a prática intervencionista educacional na saúde, não se faz apenas pelo modelo preventivo ou tradicional também chamado de "velha" saúde pública, mas também pela consideração do sistema como um todo, levando em conta além de hábitos saudáveis individualistas os princípios holísticos da "nova" saúde pública, considerando a influência social na determinação, estruturação, e padronização das doenças; ressaltando aqui as diversidades socioeconômicas, culturais e educacionais da população ${ }^{23}$.

Esta abordagem do déficit no sucesso em minimização de doenças por estratégias preventivas e educacionais, sem levar em consideração o social, as discrepâncias entre a sociedade federativa já se mostraram falha, como têm sido demonstrados para outra doença relacionada ao contágio viral, a AIDS. Uma pesquisa relacionou que mesmo em indivíduos bem informados, muitos não adotam os comportamentos preventivos que são sugeridos e prescritos ${ }^{24}$.

Assim uma preocupação se emerge para ser levada em consideração: a gri- 
pe provocada pelo vírus da Influenza A - H1N1 é apenas um exemplo que conseguiu abalar as estruturas em gestão de saúde pública mundial pela sua alta capacidade de propagação, por meio de um processo altamente valorizado mundialmente, a globalização. Recursos primários que poderiam servir de ferramenta contra esta propagação não foram eficazes, sobrecarregando assim um segundo nível de contenção, o tratamento e a cura da doença.

Os dados obtidos neste trabalho permitem ainda avaliar que embora haja uma flutuação no conhecimento da população quanto à gripe provocada pelo vírus da Influenza A - H1N1, esta variação não é significativa quando comparada às diferentes faixas etárias e/ou mesmo os diferentes gêneros. Este representa um fato tranquilizador caso as afirmativas fossem positivas quanto à propagação ao conhecimento e as suas habilidades em execução da prevenção à doença, porém não foi o que observamos. Desta forma, a prerrogativa que se deve considerar é o emergir das políticas em saúde pública de forma eficiente e capaz de promover a manutenção à saúde e a minimização de gastos $^{25}$, levando em consideração as desigualdades geográficas e sociais na utilização de serviços de saúde no país como fatores preponderantes no nivelamento para uma boa prática em educação aplicada ao desenvolvimento e manutenção à saúde $26-27$.

\section{Conclusão}

Esta abordagem permite ressaltar como um indicador a divergência entre os objetivos propostos na gestão à saúde pública, mais pontualmente a uma doença amplamente disseminada, a gripe, com os resultados do conhecimento da população regional acerca desta doença, demonstrando uma fragilidade populacional a uma possível propagação de uma nova pandemia. 1

\section{Referências}

1. Carneiro M, Trench FJP, Waib LF, Pedro FL, Motta F. Influenza H1N1 2009: revisão da primeira pandemia do século XXI. Revista AMRIGS. 2010;54(2):206-13.

2. Sakai M, Guedes $D$, José $E$, Rocha RL, Reggiani M, Lança SB, et al. Infecção pelo vírus Influenza pandêmico (H1N1) 2009. Revista Médica de Minas Gerais. 2010;20(4):578-93.

3. Medina RA. 1918 influenza virus: 100 years on, are we prepared against the next influenza pandemic? Nature Reviews Microbiology. 2018;16(2):61-2.

4. Gagnon A, Acosta E, Hallman S, Bourbeau R, Dillon LY, Ouellette N, et al. Pandemic Paradox: Early Life H2N2 Pandemic Influenza Infection Enhanced Susceptibility to Death during the 2009 H1N1 Pandemic. mBio. 2018;9(1):e02091-17.

5. Wilson KE, Wood SM, Schaecher KE, Cromwell KB, Godich J, Knapp $\mathrm{MH}$, et al. Nosocomial outbreak of influenza A H3N2 in an inpatient oncology unit related to health care workers presenting to work while ill. American Journal of Infection Control. 2019;pii: S0196-6553(18):310691.

6. Campos HR, Kazikawa GT, de Souza Pinheiro JP, dos Santos FAL, Isoton DA. Análise epidemiológica da pandemia pelo Influenza A (H1N1) no Brasil nos anos de 2009 a 2010. TCC. São Paulo; Universidade de São Paulo; 2018.

7. Brandão CR, Brandão C. Educação? Educações: aprender com o Índio [Internet]. 1995 [cited 2018 Mar 12]. Avalaible from: http://www2.unifesp.br/centros/cedess/CDRom/ativprati2.htm.

8. Moura SM, Belgalll F, SilvalV PM, RodriguesV AT. Promoção da saúde: desafios revelados em práticas exitosas. Revista de Saúde Pública. 2014;48(1):76-85. 9. Brug J, Aro AR, Richardus JH. Risk perceptions and behaviour: towards pandemic control of emerging infectious diseases. International Journal of Behavioral Medicine. 2009;16(3):3-6.

10. Cerbino Neto J. Pandemia de influenza no Brasil: epidemiologia, tratamento e prevenção da influenza A (H1N1). Rio de Janeiro:Escola Nacionald de Saúde Pública Sergio Arouca; 2012.

11. Respiratória T. Características dos casos notificados de Influenza A/ H1N1. Revista de Saúde Pública. 2009;43(5):900-4.

12. Rossetto ÉV. Estudo descritivo da pandemia de Influenza A (H1N1) pdm09 no Brasil, 2009-2010. Dissertação. São Paulo; Universidade de São Paulo; 2014.

13. Luna EJdA, Gattás VL, Campos SRdSL. Efetividade da estratégia brasileira de vacinação contra influenza: uma revisão sistemática. Epidemiologia e Serviços de Saúde. 2014;23(3):559-75.
14. Lenzi L, de Mello ÂM, da Silva LR, Grochocki MHC, Pontarolo R. Influenza pandêmica A (H1N1) 2009: fatores de risco para o internamento. Jornal Brasileiro de Pneumologia. 2012;38(1):57-65.

15. Temporão JG. 0 enfrentamento do Brasil diante do risco de uma pandemia de influenza pelo vírus A (H1N1). Epidemiologia e Serviços de Saúde. 2009;18(3):201-4.

16. Ayres J. 0 cuidado, os modos de ser (do) humano e as práticas de saúde. Saúde e sociedade. 2004;13(3):16-29.

17. Berbel $D B$, Rigolin CCD. Educação e promoção da saúde no Brasil através de campanhas públicas. Revista Brasileira de Ciência e Tecnologia Social. 2011;2(1):25-38.

18. Falkenberg MB, Mendes TdPL, Moraes EPd, Souza EMd. Educação em saúde e educação na saúde: conceitos e implicações para a saúde coletiva. Ciência e Saúde Coletiva. 2014;19(3):847-52.

19. Prodanov CC, Freitas EC. Metodologia do trabalho científico: métodos e técnicas da pesquisa e do trabalho. 2. ed. Novo Hamburgo: Feevale, 2013.

20. Silva EL, Menezes EM. Metodologia da pesquisa e elaboração de dissertação. 3. ed. rev. atual. - Florianópolis: Laboratório de Ensino a Distância da UFSC, 2001.

21. Zanella LCH. Metodologia de pesquisa. 2. ed. rev. atual. - Florianópolis: Departamento de Ciências da Administração/UFSC, 2011.

22. Sánchez AIM, Bertolozzi MR. Pode o conceito de vulnerabilidade apoiar a construção do conhecimento em Saúde Coletiva. Ciência \& Saude Coletiva. 2007;12(2):319-24.

23. da Costa Silva CM, de Castro Meneghim M, Pereira AC, Mialhe FL. Educação em saúde: uma reflexão histórica de suas práticas. Ciência \& Saúde Coletiva. 2010;15(5):2539-50.

24. Oliveira DL. A 'nova'saúde pública e a promoção da saúde via educação: entre a tradição e a inovação. Revista Latino-americana de Enfermagem. 2005;13(3):423-31.

25. Oliveira DLLCD. Brazilian adolescent women talk about HIV/AIDS risk: reconceptualizing risky sex-what implications for health promotion? London: Institute of Education, University of London; 2001.

26. Zucchi P, Del Nero C, Malik AM. Gastos em saúde: os fatores que agem na demanda e na oferta dos serviços de saúde. Saúde e Sociedade. 2000;9(1/2):127-50.

27. Travassos C, Viacava F, Fernandes C, Almeida CM. Desigualdades geográficas e sociais na utilização de serviços de saúde no Brasil. Ciência \& Saúde Coletiva. 2000;5(1):133-49. 